\title{
Scattering of Bunched Fractionally Charged Quasiparticles
}

\author{
Y. C. Chung, M. Heiblum, and V. Umansky \\ Braun Center for Submicron Research, Department of Condensed Matter Physics, Weizmann Institute of Science, Rehovot \\ 76100, Israel
}

(September 22, 2018)

\begin{abstract}
The charge of fractionally charged quasiparticles, proposed by Laughlin to explain the fractional quantum Hall effect (FQHE), was recently verified by measurements. Charge $q=e / 3$ and $e / 5(e$ is the electron charge), at filling factors $\nu=1 / 3$ and $2 / 5$, respectively, were measured. Here we report the unexpected bunching of fractional charges, induced by an extremely weak backscattering potential at exceptionally low electron temperatures $(T<10 \mathrm{mK})$ - deduced from shot noise measurements. Backscattered charges $q=\nu e$, specifically, $q=e / 3, q=2 e / 5$, and $q<3 e / 7$, in the respective filling factors, were measured. For the same settings but at an only slightly higher electron temperature, the measured backscattered charges were $q=e / 3, q=e / 5$, and $q=e / 7$. In other words, bunching of backscattered quasiparticles is taking place at sufficiently low temperatures. Moreover, the backscattered current exhibited distinct temperature dependence that was correlated to the backscattered charge and the filling factor. This observation suggests the existence of 'low' and 'high' temperature backscattering states, each with its characteristic charge and characteristic energy.
\end{abstract}

PACS numbers: 73.43.Fj, 71.10.Pm, 73.50.Td

While Laughlin's argument, explaining the fractional quantum Hall effect (FQHE) [1], is useful in predicting the charge of the quasiparticles for fractional filling factors of the type $\nu=1 /(2 p+1)$, the composite Fermion $(\mathrm{CF})$ model [2] is helpful in more general filling factors, such as $\nu=n /(2 n p+1)$, with $p$ and $n$ integers. The predicted quasiparticle charge is always $e^{*}=e /(2 n p+1)$. For $p=1,2$, and 3 and $n=1$, or alternatively for $\nu=1 / 3,2 / 5$ and $3 / 7$, we expect $q=e / 3, e / 5$ and $e / 7$, respectively. Indeed recent quantum shot noise measurements confirmed these predictions at $\nu=1 / 3$ and $2 / 5$. The shot noise, in turn, resulting from weak backscattering of quasiparticles by a quantum point contact (QPC), was measured at electron temperatures $30 \sim 80 \mathrm{mK}$, led to charges $e / 3$ at $\nu=1 / 3$ [3] and $e / 5$ at $\nu=2 / 5$ [4] - as expected. Here we report on shot noise measurements in the extreme limits of: (a) weak backscattering $(r \sim 2 \%)$, where backscattering events are so rare assuring their independence, and (b) extremely low electron temperatures $\left(T_{\min } \sim 9 \mathrm{mK}\right)$. In this regime of a barely perturbed electron system we measured, surprisingly, shot noise corresponding to backscattered charges $q=\nu e$, namely, $q=e / 3,2 e / 5$ and $\sim 3 e / 7$ at $\nu=1 / 3$, $2 / 5$ and $3 / 7$, respectively. In other words, backscattering in this regime is that of correlated $p$ quasiparticles.

Measurements were conducted in a high mobility lowdensity two-dimensional electron gas (2DEG), embedded in a GaAs-AlGaAs heterojunction. The magnetic field was set well within the conductance plateaus of the FQHE. For example, the magnetic field at $\nu=1 / 3$ was $B \sim 14.26 T$ - near the center of the $g_{Q}=e^{2} / 3 h$ plateau (Fig. 1(a)). A QPC type potential, induced in the 2DEG with two biased metallic gates deposited on the surface of the heterojunction, served as a controlled backscattering potential. A multiple-terminal-configuration (Fig. 1(b)) was employed in order to keep the input and output differential conductance constant, $g=g_{Q}$ - independent of the transmission of the QPC [5]. The differential conductance was measured with a $3 \mathrm{~Hz} \mathrm{AC}, 0.5 \mu \mathrm{V}$ RMS, excitation voltage superimposed on a DC bias that was restricted to the linear regime of the QPC. The spectral density of the noise, $S$, was measured as function of DC current at a center frequency $1.4 \mathrm{MHz}$ and bandwidth $\sim 30 \mathrm{kHz}$ (determined by a LC resonant circuit; see Refs. 3 and 4 for more details). A low noise cryogenic preamplifier, in the vicinity of the sample, amplified the voltage fluctuations in terminal A, followed by an amplifier and a spectrum analyzer at ambient temperature, measuring the RMS fluctuations at $1.4 \mathrm{MHz}$. The temperature of the electrons was determined by measuring the equilibrium noise, $S=4 k_{B} T g$, with $k_{B}$ the Boltzman constant. Shot noise was determined by subtracting the current independent noise from the total noise signal.

Figure 1(c) shows typical differential conductance curves of a QPC at bulk filling $\nu=1 / 3$. Measurements were conducted at the lowest electron temperatures $T \sim 9 \mathrm{mK}$ for different backscattering potential strengths (controlled by the QPC gates voltage $V_{g}$ ). Even a relatively weak backscattering potential, with high voltage transmission $t=g / g_{Q} \sim 0.7(r \sim 0.3)$, lead to rather strong backscattering near zero applied voltage. Moreover, both the voltage and temperature dependence of the differential conductance were positive - qualitatively agreeing with the prediction of the chiral Luttinger liquid (CLL) model [6-9]. However, when the QPC potential was tuned even weaker this dependence reversed sign (see Figs. 1(c) and 1(d)). We concentrate now on the limiting case, namely, the extremely weak backscattering regime, 
with the temperature dependence of the backscattered current for $r \sim 0.03$ shown in Fig. 1(d). A distinct positive slope over a decade of the current is seen in the $\log \left(I_{B}\right)$ vs. $\log (T)$ characteristic, with $I_{B}$ the backscattered current.
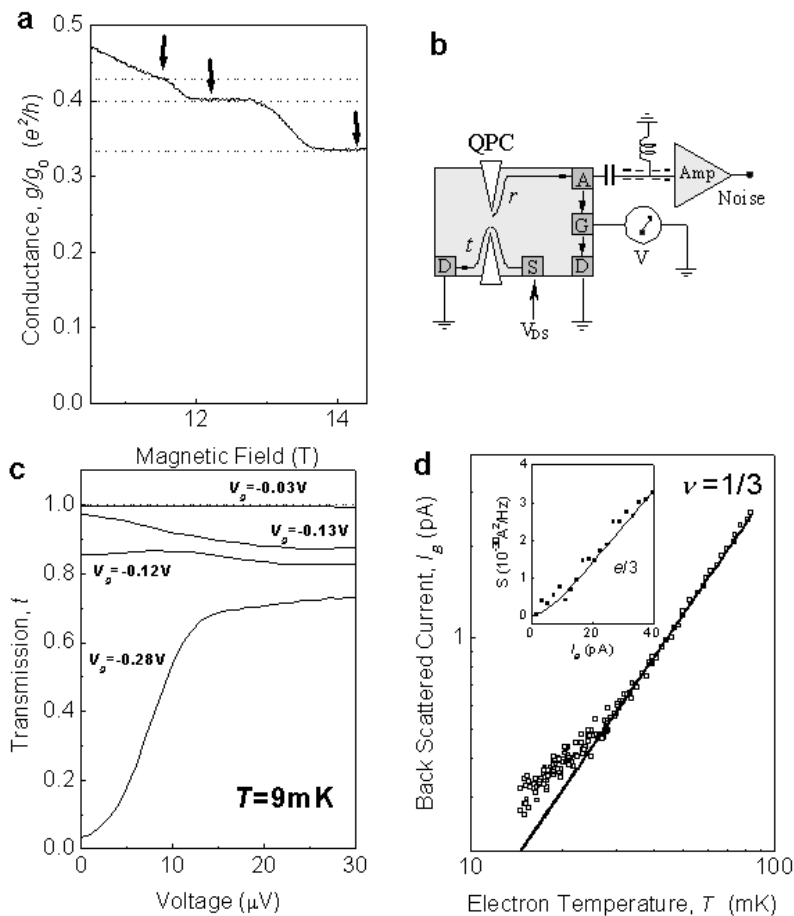

FIG. 1. (a) Quantum Hall conductance as a function of magnetic field. Filling factors were established by the pointed out magnetic fields. Similar results were obtained at different magnetic fields around the middle of the conductance plateau. (b) The measurement set up of shot noise and differential conductance. The noise generated by the QPC passed through a resonant circuit tuned to $1.4 \mathrm{HMz}$ and amplified by a cryogenic amplifier. This small capacitance at A allowed only the high frequency component through. The multiple-terminal geometry kept the conductance seen from S and A constant. (c) Typical dependence of the transmission coefficient on bias voltage for different QPC gate voltage, at $\nu=1 / 3$. When the QPC is very weakly pinched off $\left(V_{g}=-0.03 \mathrm{~V}\right)$ the transmission has a very weak negative dependence on the applied bias - oppositely to a CLL. (d) The backscattered current as a function of electron temperature with $\mathrm{AC} 10 \mu \mathrm{V}$ RMS is applied. The curve can be fitted with a single slope. Inset: The shot noise generated by a very weakly pinched off QPC $(t \sim 0.97)$ at a filling factor $\nu=1 / 3$ and electron temperature of $9 \mathrm{mK}$. Noise is classical and quasiparticle charge is $e / 3$.

We turn now to shot noise measurements. The low temperature quantum shot noise of partitioned particles in the CLL regime was predicted $[6,7]$ and later found $[8,9]$ to be highly non-classical (non-Poissonian). This is expected since backscattering of quasiparticles is correlated and energy dependent. However, when backscattering events are very rare and the tempera- ture is finite, it is expected that scattering events are stochastic with a resultant classical-like shot noise [10]. Indeed the measured spectral density of the shot noise, $S$, shown in the inset of Fig. 1(d), is classical-like. The solid line is the expected shot noise due to stochastic scattering of independent particles at $T=9 \mathrm{mK}$ and charge $q=e / 3$ [11]. It depends on $V, q, t$, and $T$ via $S=4 k_{B} T g+2 q I_{B} t \cdot \Theta(T, V)$, with $\Theta(T, V)=$ $\operatorname{coth}\left(q V /\left(2 k_{B} T\right)\right)-2 k_{B} T /(q V), I_{B}=V g_{Q}(1-t)$, and $g_{Q}=\nu e^{2} / h$. Here, for $q V \gg k_{B} T, \Theta(T, V) \sim 1$ and the dependence of $S$ on $I_{B}$ is linear, and for $q V \ll k_{B} T$ the Johnson-Nyquist thermal noise dominates. Note that at $T \sim 0$ and $t \rightarrow 1$, as in our case, $S \simeq 2 q I_{B}$. The excellent agreement between experiment and prediction proves that scattering events of $e / 3$ quasiparticles are independent down to the lowest temperatures provided that the backscattering potential is extremely weak.
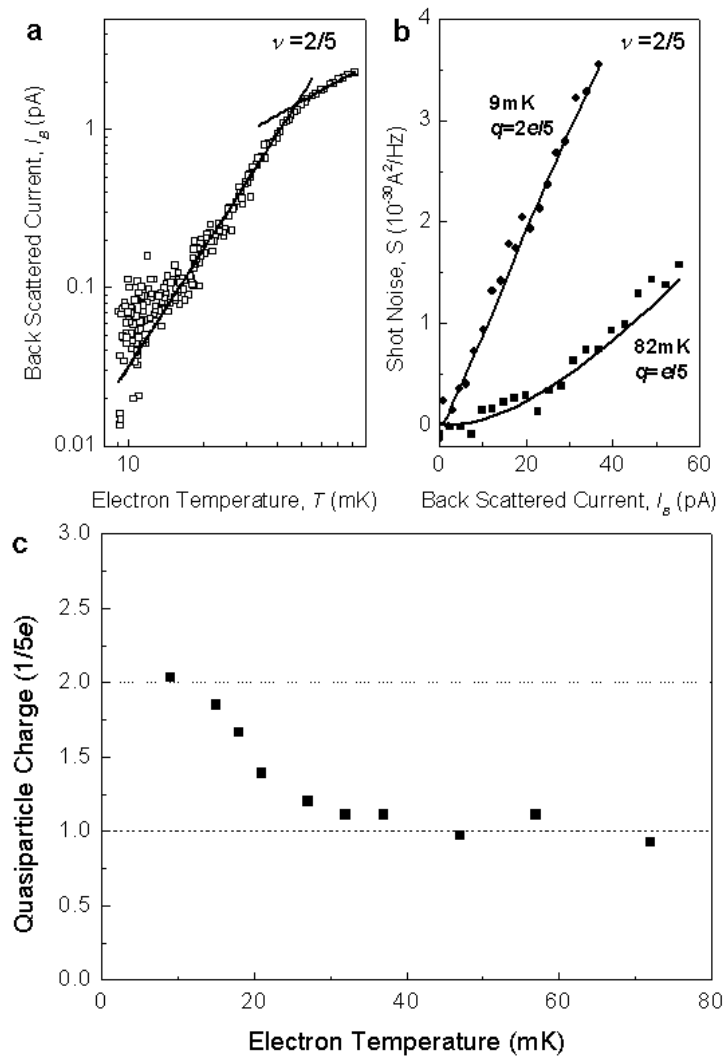

FIG. 2. (a) Backscattered current as function of the electron temperature at a filling factor $\nu=2 / 5$. Two distinct slopes are observed with a transition temperature of about $45 \mathrm{mK}$. (b) Shot noise at two different temperatures. The backscattered quasiparticle charge is $2 e / 5$ at $9 \mathrm{mK}$ and $e / 5$ at $82 \mathrm{mK}$. The QPC was set to reflect some $2 \%$ of the impinging current at the two temperatures. (c) The temperature dependence of the scattered charge when QPC was set to reflect some $2 \%$ of the impinging current. 
We now study the regime of extremely weak backscattering at $p=2$, namely, electron filling factor $\nu=2 / 5$. The magnetic field was tuned to $B=12.2 \mathrm{~T}$ within the $g_{Q}=(2 / 5) e^{2} / h$ plateau (see Fig. $\left.1(\mathrm{a})\right)$ and the QPC to $r \sim 0.02$. Note that the general features seen in Fig. 1(c) at $\nu=1 / 3$ are also found at $\nu=2 / 5$. We measured the temperature dependence of the backscattered current, as shown in Fig. 2(a), and find this time two distinct slopes in $\log \left(I_{B}\right)$ vs. $\log (T)$ - with a crossover at $T \sim 45 \mathrm{mK}$. We then measured the shot noise at different electron temperatures and found it, again, to be classical-like in all temperatures (see in Fig. 2(b) two extreme examples). When determining the charge in a most general filling factor one has to rely on the CF model. According to that model the reflected current, carrying the noise, is that of CFs in the $2^{\text {nd }}$ Landau level (LL), namely $p=2$, with the $e / 3$ quasiparticles (in the $1^{\text {st }}$ LL) being fully transmitted without contributing to the shot noise [4]. Hence, one can define an effective transmission coefficient of $2^{\text {nd }} \mathrm{LL}$ $\mathrm{CFs}, t_{\text {eff }}=\left(t \cdot g_{2 / 5}-g_{1 / 3}\right) /\left(g_{2 / 5}-g_{1 / 3}\right)=6 t-5$, which is smaller than the bare transmission $t$. However, when $t$ is very close to unity $t_{\text {eff }} \sim t$, and the determination of $q$ is not sensitive to the exact value of $t$. The two solid lines in Fig. 2(b), agreeing with the data, are the calculated shot noise according to the expression above with charges $q=2 e / 5$ at $T \sim 9 \mathrm{mK}$ and $q=e / 5$ at $T \sim 82 \mathrm{mK}$ (with electron temperatures determined independently). While the scattered charge at high temperature $q=e / 5$, had been verified before [4], the scattered charge at low temperature $q=\nu e=2 e / 5$, was unexpected. Figure $2(\mathrm{~d})$ shows the charge evolution as the temperature is being increased in the range $9 \mathrm{mK}<T<50 \mathrm{mK}$. Most of the change takes place over a $20 \mathrm{mK}$ range. In comparison, temperature dependence measurements were conducted in a separately patterned Hall bar. While the $\nu=1 / 3$ conductance plateau remained unaffected at this temperature range and the longitudinal resistance $R_{x x}$ increased with temperature, but with dependence quite different than that of $I_{B}$ from the QPC.

Does such unexpected bunching take place also in higher $\mathrm{CF}$ filling factors $p=3$, namely, at $\nu=3 / 7$ ? Because of the relatively weak magnetic field at the $\nu=3 / 7$ $(\mathrm{B}=11.5 \mathrm{~T})$ the many-body energy gap required to establish the $g_{Q}=(3 / 7) e^{2} / h$ plateau is rather small. Consequently, the plateau is barely established even at the lowest temperature (see Fig. 2(a)) and there is a finite backscattered current through the bulk (some 0.5\%) and the minimum longitudinal resistance $R_{x x}>0$. Inducing a very weak QPC potential in the $2 \mathrm{DEG}$ increased the backscattered current and produced a measurable shot noise, as seen in Fig. 3. Using the effective transmission (that is more sensitive here to the bare $t$ ) the fitted charge at the lowest temperature was extremely sensitive to minute variations in the electron temperature and seems to hover in the range $(2 \sim 2.5) e / 7$. Warming the electrons to $T \geq 27 \mathrm{mK}$ lowered significantly the shot noise and established firmly a quasiparticle charge of $e / 7$. This is the first measurement of such small fractional charge. The higher scattered charge at the lowest temperature indicates again bunching of $e / 7$ quasiparticles - very much like the behavior at $\nu=2 / 5$, however, it seems that an even lower temperature than our lowest temperature $(T<9 \mathrm{mK})$ is needed to establish bunching of three $e / 7$ quasiparticles to a charge $q=3 e / 7$ as well as to achieve a perfect FQH plateau.

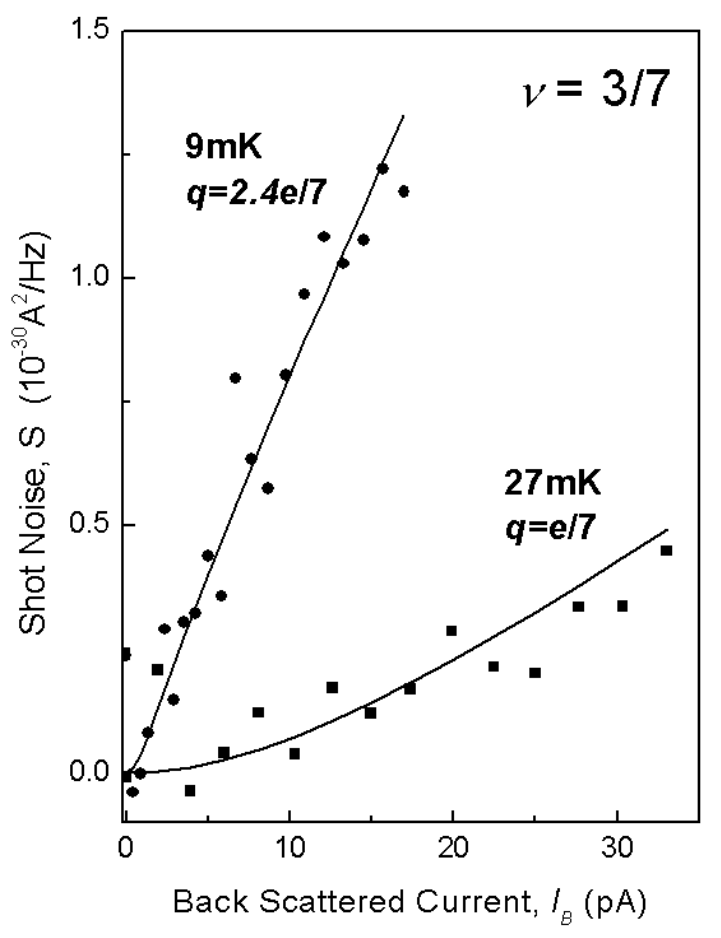

FIG. 3. Shot noise at a filling factor $\nu=3 / 7$ at two different temperatures. The backscattered quasiparticle charge is found to be around $(2 \sim 2.5) e / 7$ at $9 \mathrm{mK}$ and $e / 7$ at $27 \mathrm{mK}$. The QPC was set to reflect some $2 \%$ of the impinging current at the two temperatures.

Summarizing our results one should recall: (a) the 2DEG is rather pure with mobility $2 \times 10^{6} \mathrm{~cm}^{2} V^{-1} s^{-1}$, hence, scattering is dominated by the weak potential of the QPC; (b) the electron temperature is very low $\left(T \sim 9 \mathrm{mK}, k_{B} T \sim 0.8 \mu \mathrm{eV}\right)$, minimizing thermal noise and alleviating any ambiguity in analyzing the data; (c) the $\mathrm{QPC}$ is very open, leading to very rare backscattering events; (d) shot noise is classical-like with linear dependence of noise on current - suggesting independent scattering of quasiparticles with a specific charge; (e) pinching the QPC ever so slightly more renders both the DC current and shot noise to be highly non-linear functions of voltage - suggesting correlated scattering of quasiparticles with charge dependent on bias. These results confirm, that in a barely perturbed $2 \mathrm{DEG}$ and $T \sim 0$, a very 
weakly bound scattering state is formed, with transport dominated by independent scattering events of $p$ bunched quasiparticles with charge $q=\nu e$. In the $\mathrm{CF}$ model, rare backscattering events of simultaneous $p$ quasiparticles, one from each LL, are taking place.

Relying on the CLL model, Kane and Fisher predicted such possibility of bunching due to backscattering via to a point scatterer [12]. However, their expression for the backscattered current, $I_{B} \propto v^{2} T^{-|\alpha|}$, with $v$ an energy independent backscattering amplitude and $\alpha$ a coefficient depended on the scattered charge, suggests a decrease of the backscattered current with temperature - contradicting our data. Note though that since our QPC is almost fully open, its potential is expected to be rather smooth and shallow with energy dependent backscattering amplitude, $v=v(T, V)$. This dependence might dominate the behavior of the backscattered current leading to our result. Still, we stress, that our observations were reproducible among samples with different QPCs and different cooling cycles; hence, we believe that it is not sensitive to the details of the QPC potential.

It should be noted that our observed bunching at low temperatures is quite different from the already observed bunching by strong backscattering potentials (at $\nu=1 / 3$, bunching of quasiparticles leads to electron scattering) $[5,8,13]$. In the latter case the FQHE state does not exist in the barrier region, hence preventing the existence of elementary quasiparticles - forcing the quasiparticles to bunch to an electron. Here, however, the FQHE state is hardly perturbed in the barrier region, still allowing the existence of elementary quasiparticles. Hence, the spontaneous bunching of $p$ quasiparticles is possibly related to their fractional statistics, namely, their partly Bosonic nature, encouraging them to bunch upon scattering.
We thank C. Kane and A. Stern for helpful discussions and D. Mahalu for the submicron lithography. The work was partly supported by the Israeli Academy of Science and by the German-Israeli Foundation (GIF).

[1] R. B. Laughlin, Phys. Rev. Lett. 50, 1395 (1983).

[2] O. Heinonen (Ed.), Composite Fermions: A unified View of the Quantum Hall Regime, World Scientific, Singapore (1998).

[3] R. de-Picciotto, M. Reznikov, M. Heiblum, V. Umansky, G. Bunin, and D. Mahalu, Nature (London) 389, 162 (1997). ;L. Saminadayar, D. C. Glattli, Y. Jin, and B. Etienne, Phys. Rev. Lett, 79, 2526 (1997).

[4] M. Reznikov, R. de-Picciotto, T. G. Griffiths, M. Heiblum, and V. Umansky, Nature (London) 399, 238 (1999).

[5] E. Comforti, Y. C. Chung, M. Heiblum V. Umansky and D. Mahalu, Nature (London) 416, 515 (2002).

[6] X. G. Wen, Phys. Rev, B 44, 5708 (1991).

[7] P. Fendley, and H. Saleur, Phys. Rev. B 52, 8934 (1995).

[8] Y. C. Chung, M. Heiblum, Y. Oreg, V. Umansky, and D. Mahalu, (to be published in Phys. Rev. B).

[9] D. C. Galattli, V. Rodriguez, H. Perrin, P. Roche, and B. Etienne, Physica. (Amsterdam) E 6, 22 (2000).

[10] C. L. Kane, and M. P. A. Fisher, Phys. Rev. Lett. 72, 724 (1994).

[11] T. Martin, and R. Landauer, Phys Rev. B 46, 12485 (1992).

[12] C. L. Kane, and M. P. A. Fisher, Phys. Rev. B 51, 13449 (1995).

[13] T. G. Griffths, E. Comforti, M. Heiblum, A. Stern and V. Umansky, Phys. Rev. Lett, 85, 3918 (2000). 